\title{
The Potential of Leptonic Minimal Flavour Violation
}

\author{
Rodrigo Alonso \\ Departamento de Física Teórica, Universidad Autónoma de Madrid and \\ Instituto de Física Teórica IFT-UAM/CSIC, Cantoblanco, 28049 Madrid, Spain
}

\begin{abstract}
We explore the possibility of spontaneous lepton flavour symmetry breaking in the context of Minimal Flavour Violation and within a minimal and predictive See-Saw model. In this context the Yukawa couplings are the vacuum expectation values of scalar fields transforming under the flavour group. The scalar potential giving rise to the acquisition of the vev of the scalar fields is derived and analyzed. In the minimization the Majorana character of neutrino masses plays a distinctive role, absent for the quark case.
\end{abstract}

Keywords: Flavour, Neutrino Masses, Mixing angles, Majorana Fermions

\section{Introduction}

The recent discovery of a bosonic particle at LHC $([1,2])$ adds another piece of support to the astonishingly well consolidated building of the Standard Model (SM). Still some aspects of the SM raise yet unanswered questions. One long standing puzzle is that of explaining in more fundamental terms the flavour structure within the SM, with masses spanning orders o magnitude and mixing angles being perturbative for quarks but close to maximal for leptons. In this context the Minimal Flavour Violation (MFV) hypothesis, applicable for both quarks $[3,4,5]$ and leptons $[6,7,8]$ offers a predictive scheme based on symmetries. This symmetry argument in the quark sector was taken further and the spontaneous symmetry breaking necessary to account for masses and mixing has been studied $[9,10,11,12]$. Here the lepton sector will be addressed in the same context, with the distinctive feature of a possible Majorana character, a study carried out in [13]. For the study we will use a predictive seesaw model $[14,15]$ with two right-handed neutrinos, satisfying the hypothesis of MFV.

Email address: rodrigo.alonso@uam.es (Rodrigo Alonso)

\section{The model}

The Lagrangian reads

$$
\begin{aligned}
-\mathcal{L}_{\text {mass }}= & \bar{\ell}_{L} \phi Y_{E} E_{R}+\bar{\ell}_{L} \tilde{\phi}\left(Y N+Y^{\prime} N^{\prime}\right)+ \\
& +\Lambda \overline{N^{\prime}} N^{c}+\text { h.c. }
\end{aligned}
$$

where $\ell_{L}$ denotes the left-handed (LH) lepton doublet, $E_{R}$ denotes the RH charged lepton fields (flavour indices are implicit) and $\phi$ denotes the Higgs doublet, with $\tilde{\phi}=i \tau_{2} \phi^{*}$ and $\langle\phi\rangle \equiv \mathrm{v} / \sqrt{2}$ the electroweak vev, with $\mathrm{v}=246 \mathrm{GeV}$. The model contains only two heavy neutrinos, $N$ an $N^{\prime}$, and in consequence one light neutrino remains massless -an open possibility to this date- and there is only one physical Majorana phase. $\Lambda$ is a Majorana scale, while the Yukawa couplings for charged leptons $Y_{E}$, and for neutrinos $Y$ and $Y^{\prime}$, are a matrix and vectors in flavour space, respectively. The Lepton Number (LN) symmetry is violated by the simultaneous presence of $Y, Y^{\prime}$ and $\Lambda$. The light neutrino matrix reflects these properties and takes the typical form in type I See-Saw models:

$$
\bar{v}_{L} \frac{\mathrm{v}^{2}}{2 \Lambda}\left(Y Y^{\prime T}+Y^{\prime} Y^{T}\right) v_{L}^{c}+\text { h.c. }
$$

where $\Lambda \gg v$ has been assumed. 
This model can accommodate small neutrino masses in the $\mathrm{LN}$ conservation limit $\left(y^{\prime} \ll y\right)$ yet still observable flavour violating processes are present for $\Lambda \sim \mathrm{TeV}[15$, $16,17,18,19]$. Without loss of generality, we work in the basis in which the charged lepton Yukawa matrix is diagonal. Denoting by $Y_{v}$ the matrix constructed out of the two vectors $Y$ and $Y^{\prime} ; Y_{v} \equiv\left(Y, Y^{\prime}\right)$, the Yukawa couplings may be described by [15]

$$
Y_{E}=\left(\begin{array}{ccc}
y_{e} & 0 & 0 \\
0 & y_{\mu} & 0 \\
0 & 0 & y_{\tau}
\end{array}\right), \quad Y_{v}=U f_{m_{v}}\left(\begin{array}{cc}
-i y & i y^{\prime} \\
y & y^{\prime}
\end{array}\right) \text {, }
$$

where $y \equiv \sqrt{(Y)^{\dagger} Y}$ and $y^{\prime} \equiv \sqrt{\left(Y^{\prime}\right)^{\dagger} Y^{\prime}}$. Here $U$ denotes the PMNS mixing matrix: using the PDG notation, $U$ is written as the product of three rotations and a matrix containing the Majorana phases, $U=$ $R_{23}\left(\theta_{23}\right) R_{13}\left(\theta_{13}, \delta\right) R_{12}\left(\theta_{12}\right) \Omega . \quad \Omega$ can be parametrised as $\Omega=\operatorname{diag}\left\{1, e^{i \alpha}, e^{-i \alpha}\right\}$ for normal hierarchy $(\mathrm{NH})$ and $\Omega=\operatorname{diag}\left\{e^{i \alpha}, e^{-i \alpha}, 1\right\}$ for inverted hierarchy (IH). The term $f_{m_{v}}$ is a matrix function of neutrino masses: for the two possible hierarchies it is defined as:

$$
\begin{gathered}
\mathrm{NH}: \quad f_{m_{v}}=\frac{1}{\sqrt{m_{v_{2}}+m_{v_{3}}}}\left(\begin{array}{cc}
0 & 0 \\
\sqrt{m_{v_{2}}} & 0 \\
0 & \sqrt{m_{v_{3}}}
\end{array}\right), \\
\left(m_{v_{2}}\right)^{2}=\Delta m_{\text {sol }}^{2}, \quad\left(m_{v_{3}}\right)^{2}=\Delta m_{\text {atm }}^{2}+\Delta m_{\text {sol }}^{2}, \\
\mathrm{IH}: \quad f_{m_{v}}=\frac{1}{\sqrt{m_{v_{1}}+m_{v_{2}}}}\left(\begin{array}{cc}
\sqrt{m_{v_{1}}} & 0 \\
0 & \sqrt{m_{v_{2}}} \\
0 & 0
\end{array}\right), \\
\left(m_{v_{1}}\right)^{2}=\Delta m_{\text {atm }}^{2}-\Delta m_{\text {sol }}^{2}, \quad\left(m_{v_{2}}\right)^{2}=\Delta m_{\text {atm }}^{2} .
\end{gathered}
$$

\section{The Scalar Potential}

In the spirit of MFV, the flavour symmetry of the model is identified as the extended symmetry arising in the limit of vanishing Yukawa couplings:

$$
\mathcal{G}_{f l} \sim S U(3)_{\ell_{L}} \times S U(3)_{E_{R}} \times O(2)_{N},
$$

with the special unitary groups associated to complex rotations of same-charge SM fields and the orthogonal group composed out of a phase rotation opposite for $N$ with respect to $N^{\prime}\left(N e^{i \phi}, N^{\prime} e^{-i \phi}\right)$ times their exchange.

For this symmetry to be formally conserved in the presence of the Yukawa couplings the latter should be assigned transformation properties under $\mathcal{G}_{f l}$,

$$
Y_{E} \sim(3, \overline{3}, 1), \quad \tilde{Y}_{v} \sim(3,1,2) .
$$

The hypothesis of MFV then allows to construct a flavour-predictive effective field theory [5] which in this case can be reproduced to all orders as the complete model is at disposal.

Let us now advance in the symmetry reasoning. Consider the flavour symmetry as an exact symmetry of the full Lagrangian, although, necessarily broken at low energies. Mimicking the Higgs mechanism, some scalar fields with transformation properties under this symmetry (dubbed flavons) must be responsible for the breaking. In the simplest realization these fields are no other than the Yukawa couplings upgraded to scalar fields, whose vev will therefore shall be proportional to the latter:

$$
\begin{gathered}
\boldsymbol{y}_{E} \sim(3, \overline{3}, 1), \quad \boldsymbol{y}_{v} \sim(3,1,2) . \\
\frac{\left\langle\boldsymbol{y}_{E}\right\rangle}{\Lambda_{f l}} \equiv Y_{E}, \quad \frac{\left\langle\boldsymbol{y}_{v}\right\rangle}{\Lambda_{f l}} \equiv Y_{v} .
\end{gathered}
$$

The scalar potential now will be build up of flavour invariant arrangements of these fields, they are; to the renormalizable level:

$$
\begin{gathered}
\operatorname{Tr}\left(\boldsymbol{y}_{E} \boldsymbol{y}_{E}^{\dagger}\right), \quad \operatorname{Tr}\left(\boldsymbol{y}_{v} \boldsymbol{y}_{v}^{\dagger}\right), \quad \operatorname{det}\left(\boldsymbol{y}_{E}\right), \\
\operatorname{Tr}\left(\boldsymbol{y}_{E} \boldsymbol{y}_{E}^{\dagger}\right)^{2}, \quad \operatorname{Tr}\left(\boldsymbol{y}_{E} \boldsymbol{y}_{E}^{\dagger} \boldsymbol{y}_{v} \boldsymbol{y}_{v}^{\dagger}\right), \\
\operatorname{Tr}\left(\boldsymbol{y}_{v} \mathcal{Y}_{v}^{\dagger}\right)^{2}, \quad \operatorname{Tr}\left(\boldsymbol{y}_{v} \sigma_{3} \boldsymbol{Y}_{v}^{\dagger}\right)^{2}
\end{gathered}
$$

The presence of the determinant can be forbidden if the flavour symmetry is extended to contain overall $U(1)$ rotations of same-charge fields [13]. The scalar potential $V$ for the flavon fields is then:

$$
\begin{aligned}
V= & -\mu^{2} \cdot \mathbf{X}^{2}+\left(\mathbf{X}^{2}\right)^{\dagger} \lambda \mathbf{X}^{2}+\left(\mu_{D} \operatorname{det}\left(\boldsymbol{y}_{E}\right)+\text { h.c. }\right)+ \\
& +\lambda_{E} \operatorname{Tr}\left(\boldsymbol{y}_{E} \boldsymbol{Y}_{E}^{\dagger}\right)^{2}+g \operatorname{Tr}\left(\boldsymbol{y}_{E} \boldsymbol{Y}_{E}^{\dagger} \boldsymbol{y}_{v} \boldsymbol{Y}_{v}^{\dagger}\right)+ \\
& +h \operatorname{Tr}\left(\boldsymbol{y}_{v} \boldsymbol{Y}_{v}^{\dagger}\right)^{2}+h^{\prime} \operatorname{Tr}\left(\boldsymbol{y}_{v} \sigma_{3} \boldsymbol{Y}_{v}^{\dagger}\right)^{2} .
\end{aligned}
$$

In this equation $\mathbf{X}^{2}$ is a two-component vector defined by

$$
\mathbf{X}^{2} \equiv\left(\operatorname{Tr}\left(\boldsymbol{y}_{E} \boldsymbol{Y}_{E}^{\dagger}\right), \operatorname{Tr}\left(\boldsymbol{y}_{v}^{\dagger} \boldsymbol{y}_{v}\right)\right)^{T},
$$

$\mu^{2}$ is a real two-component vector, $\lambda$ is a $2 \times 2$ Hermitian matrix and all other coefficients are real parameters, except for $\mu_{D}$ which may be complex. The full scalar potential includes in addition Higgs- $\mathcal{Y}_{E}$ and Higgs- $\boldsymbol{y}_{v}$ cross-terms, but they do not affect the mixing pattern and will thus be obviated in what follows.

\subsection{Mixing angles and Majorana Phase}

The emphasis is here in the way this potential fixes the mixing pattern, so consider here the fermion masses fixed at their physical values and focus on the minimization on the mixing parameters. Since mixing arises from 
the misalignment in flavour space of the charged lepton and neutrino sectors, the only relevant invariant at the renormalizable level is

$$
\boldsymbol{O}_{\text {mix }} \equiv \operatorname{Tr}\left(\boldsymbol{y}_{E} \mathcal{Y}_{E}^{\dagger} \boldsymbol{y}_{v} \boldsymbol{y}_{\nu}^{\dagger}\right) \text {. }
$$

Substituting the expressions for the flavon vevs in Eq. (8), it follows that:

$$
\begin{aligned}
O_{\text {mix }}= & \frac{2 \Lambda_{f l}^{4}}{v^{2} \sum m_{v_{i}}}\left(\sigma_{y} \sum_{l, i}\left|U^{l i}\right|^{2} m_{l}^{2} m_{v_{i}}\right. \\
& \left.+\delta_{y}\left(i e^{2 i \alpha} \sum_{l, i<j}\left(U^{l i}\right)^{*} U^{l j} m_{l}^{2} \sqrt{m_{v_{i}} m_{v_{j}}}+\text { c.c. }\right)\right),
\end{aligned}
$$

with $\sigma_{y}=y^{2}+y^{\prime 2}$ and $\delta_{y}=y^{2}-y^{\prime 2}$. The first term in Eq. (12) for leptons corresponds to that for quarks (in the bifundamental representation case [12]). The second term in Eq. (12) has a strong impact on the localization of the minimum of the potential and is responsible for the different results in the quark and lepton sectors: it contains the Majorana phase $\alpha$ and therefore connects the Majorana nature of neutrinos to their mixing.

The two family case will help now see the features of the minimization process and afterwards the generalization to the three family scenario will be straightforward. The mixing invariant reads, for two families:

$$
\begin{aligned}
g O_{\text {mix }} \propto & g\left\{\left(m_{e}^{2}+m_{\mu}^{2}\right)\left(y^{2}+y^{\prime 2}\right)\left(m_{v_{2}}+m_{v_{1}}\right)+\right. \\
& +\left(m_{\mu}^{2}-m_{e}^{2}\right)\left[\left(m_{v_{2}}-m_{v_{1}}\right)\left(y^{2}+y^{\prime 2}\right) \cos 2 \theta+\right. \\
& \left.\left.+\left(y^{2}-y^{\prime 2}\right) 2 \sqrt{m_{v_{2}} m_{v_{1}}} \sin 2 \alpha \sin 2 \theta\right]\right\},
\end{aligned}
$$

where $\theta$ is the mixing angle and $\alpha$ the Majorana phase. This formula shows explicitly the relations expected on physical grounds, between the mass spectrum and nontrivial mixing: i) the dependence on the mixing angle disappears in the limit of degenerate charged lepton masses; ii) it also vanishes for degenerate neutrino masses if and only if $\sin 2 \alpha=0$; iii) on the contrary, for $\sin 2 \alpha \neq 0$ the dependence on the mixing angle remains, as it is physical even for degenerate neutrino masses; iv) the $\alpha$ dependence vanishes when one of the two neutrino masses vanishes or in the absence of mixing, as $\alpha$ becomes then unphysical.

The minimization with respect to the Majorana phase and the mixing angle leads to the relations:

$$
\begin{gathered}
\left(y^{2}-y^{\prime 2}\right) \sqrt{m_{v_{2}} m_{v_{1}}} \sin 2 \theta \cos 2 \alpha=0, \\
\operatorname{tg} 2 \theta=\sin 2 \alpha \frac{y^{2}-y^{\prime 2}}{y^{2}+y^{\prime 2}} \frac{2 \sqrt{m_{v_{2}} m_{v_{1}}}}{m_{v_{2}}-m_{v_{1}}} .
\end{gathered}
$$

The first condition predicts then that the Majorana phase is maximal, $\alpha= \pm \pi / 4$, for non-trivial mixing angle. The relative Majorana phase between the two neutrinos is therefore $2 \alpha= \pm \pi / 2$ which implies no $\mathrm{CP}$ violation due to Majorana phases. On the other hand, Eq. (22) establishes a link between the mixing strength and the type of spectrum, which indicates a maximal angle for degenerate neutrino masses, and a small angle for strong mass hierarchy. At the minimum, the value of the mixing term is:

$$
\begin{cases}\left.O_{\text {mix }}\right|_{\text {min }} \propto m_{e}^{2} m_{+}+m_{\mu}^{2} m_{-}, & g>0, \\ \left.O_{\text {mix }}\right|_{\text {min }} \propto m_{e}^{2} m_{-}+m_{\mu}^{2} m_{+}, & g<0,\end{cases}
$$

where

$$
\begin{aligned}
& m_{ \pm} \equiv a_{v} \pm \sqrt{a_{v}^{2}-c_{v}^{2}}, \\
& a_{v}=\left(m_{v_{2}}+m_{v_{1}}\right)\left(y^{2}+y^{2}\right), c_{v}=4 \sqrt{m_{v_{2}} m_{v_{1}}} y y^{\prime} .
\end{aligned}
$$

The two quantities $m_{ \pm}$are proportional to the eigenvalues of $Y_{v} Y_{v}^{\dagger}$ just like $m_{e}^{2}$ and $m_{\mu}^{2}$ with respect to $Y_{E} Y_{E}^{\dagger}$. This gives an insight in the minimization process and a rule to easily spot the mixing configuration at the minimum; the mixing pattern at the minimum is that for which the eigenvalues of the Yukawa couplings are connected one to one in increasing $(g<0)$ or decreasing $(g>0)$ order. This ordering recalls hierarchy and indeed it is related, $m_{ \pm}$are connected to the heaviest and lightest neutrino mass eigenstates, and the sign of $g$ sets the hierarchy.

Inspection of the Yukawa structure dependence in masses $y$, and $y^{\prime}$ in Eqs $(3,4)$ reveals that one nontrivial mixing angle only suffices for the diagonalization also in the three family case. This stems in the vanishing of the third neutrino mass and is specific to this model, and in turn implies that the mixing pattern of $U_{p m n s}$ cannot be fully reproduced:

$\mathrm{NH}(g<0)$ The minimum imposes $\theta_{13}=\theta_{12}=0, \alpha=$ $\pm \pi / 4$ and the the atmospheric angle is determined by Eq. (15) with the substitutions $m_{v_{1}}^{2}=\Delta m_{\text {sol }}^{2}$, $m_{v_{2}}^{2} \simeq \Delta m_{\text {atm }}^{2}$, which produce a too small value due to the hierarchical set-up.

IH $(g>0)$ In this case $\theta_{13}=\theta_{23}=0, \alpha= \pm \pi / 4$ and the substitution $m_{v_{1}}^{2} \simeq m_{v_{2}}^{2}=\Delta m_{\text {atm }}^{2}$ in Eq. (15) reveals a maximal solar angle $\theta_{12} \simeq \pi / 4$ for the quasi-degenerate neutrino spectrum [13].

\section{Conclusions}

An outstanding question is whether the strong mixing pattern found in the leptonic sector - in contrast to 
the small mixing and hierarchical spectrum of the quark sector - is related to the possible Majorana character of the neutrino fields. While this cannot be answered in general, we have shown that the hypothesis of a dynamical realization of MFV may be restrictive enough to answer it within its framework.

We have explored the possibility of a dynamical origin of the Yukawa couplings of leptons in the context of MFV and Majorana neutrinos. The simplest realization is to identify the Yukawa couplings with the vevs of some dynamical scalar fields, the flavons. The implementation of the Majorana character needs to refer to an explicit model of Majorana neutrino masses. We concentrated here in one of the simplest possible MFV models of Majorana neutrino masses: a See-Saw model with only two extra heavy singlet neutrinos with approximate lepton number symmetry [15]. We determined the corresponding scalar potential under the only requirement of SM gauge invariance and invariance under the underlying flavour symmetry and explored its minima.

The toy model with only two families demonstrates that non-trivial Majorana phases and mixing angles may be selected by the potential minima and indicates a novel connection with the pattern of neutrino masses: i) mixing angles and neutrino mass spectrum are tightly related; degenerate spectrum implies maximal angles whereas a hierarchical scheme sets vanishing angles ii) the relative Majorana phase among the two massive neutrinos is predicted to be maximal, $2 \alpha=\pi / 2$ but not $\mathrm{CP}$ violating. These novel results are intimately related with the well-known fact that, in the presence of non-trivial Majorana phases, there may be physical mixing even for degenerate neutrino masses. In consequence, the results are expected to hold as well for general fermionic See-Saw scenarios.

Upgrading the analysis to three lepton generations and under the same model assumptions, the minimum of the potential cannot correspond to the measured values of masses and mixings. This is linked to the necessary masslessness of the third left-handed neutrino in the minimal model, which imposes a fixed hierarchy with respect to the massive modes. However more freedom is expected in models with three massive neutrinos.

\section{Acknowledgments}

R. A. acknowledge partial support from the European Union FP7 ITN INVISIBLES (Marie Curie Actions, PITN- GA-2011- 289442), CiCYT support through the project FPA2009-09017, CAM partial support through the project HEPHACOS P-ESP-00346 and MICINN support through the grant BES-2010-037869. Finally the author thanks the Physics Department at Harvard University for hospitality.

[1] G. Aad et al. [ATLAS Collaboration], Phys. Lett. B 716 (2012) 1 [arXiv:1207.7214 [hep-ex]].

[2] S. Chatrchyan et al. [CMS Collaboration], Phys. Lett. B 716 (2012) 30 [arXiv:1207.7235 [hep-ex]].

[3] R. S. Chivukula and H. Georgi, Composite Technicolor Standard Model, Phys. Lett. B188 (1987) 99.

[4] L. J. Hall and L. Randall, Weak Scale Effective Supersymmetry, Phys. Rev. Lett. 65 (1990) 2939-2942.

[5] G. D'Ambrosio, G. F. Giudice, G. Isidori, and A. Strumia, Minimal Flavour Violation: an Effective Field Theory Approach, Nucl. Phys. B645 (2002) 155-187, [hep-ph/0207036].

[6] V. Cirigliano, B. Grinstein, G. Isidori, and M. B. Wise, Minimal flavor violation in the lepton sector, Nucl. Phys. B728 (2005) 121-134, [hep-ph/0507001].

[7] S. Davidson and F. Palorini, Various Definitions of Minimal Flavour Violation for Leptons, Phys. Lett. B642 (2006) 72-80, [hep-ph/0607329].

[8] R. Alonso, G. Isidori, L. Merlo, L. A. Munoz, and E. Nardi, Minimal Flavour Violation Extensions of the Seesaw, JHEP 06 (2011) 037, [hep-ph/1103.5461].

[9] A. Anselm and Z. Berezhiani, Weak Mixing Angles as Dynamical Degrees of Freedom, Nucl.Phys. B484 (1997) 97-123, [hep$\mathrm{ph} / 9605400]$.

[10] Z. Berezhiani and A. Rossi, Flavor Structure, Flavor Symmetry and Supersymmetry, Nucl.Phys.Proc.Suppl. 101 (2001) 410 420, [hep-ph/0107054].

[11] T. Feldmann, M. Jung, and T. Mannel, Sequential Flavour Symmetry Breaking, Phys. Rev. D80 (2009) 033003, [arXiv:0906.1523].

[12] R. Alonso, M. B. Gavela, L. Merlo, and S. Rigolin, On the Scalar Potential of Minimal Flavour Violation, JHEP 07 (2011) 012, [arXiv:1103.2915].

[13] R. Alonso, M. B. Gavela, D. Hernandez and L. Merlo, 'On the Potential of Leptonic Minimal Flavour Violation', Phys. Lett. B 715 (2012) 194 [arXiv:1206.3167 [hep-ph]].

[14] M. Raidal, A. Strumia, and K. Turzynski, Low-Scale Standard Supersymmetric Leptogenesis, Phys.Lett. B609 (2005) 351359, [hep-ph/0408015].

[15] M. Gavela, T. Hambye, D. Hernandez, and P. Hernandez, Minimal Flavour Seesaw Models, JHEP 0909 (2009) 038, [arXiv:0906.1461].

[16] O. Eboli, J. Gonzalez-Fraile, and M. Gonzalez-Garcia, Neutrino Masses at LHC: Minimal Lepton Flavour Violation in Type-III See-Saw, JHEP 1112 (2011) 009, [arXiv:1108.0661].

[17] D. Dinh, A. Ibarra, E. Molinaro, and S. Petcov, The $\mu \rightarrow$ $e$ Conversion in Nuclei, $\mu \rightarrow e \gamma, \mu \rightarrow 3 e$ Decays and $\mathrm{TeV}$ Scale See-Saw Scenarios of Neutrino Mass Generation, [arXiv:1205.4671].

[18] D. Aristizabal Sierra, A. Degee, and J. F. Kamenik, Minimal Lepton Flavor Violating Realizations of Minimal Seesaw Models, [arXiv:1205.5547].

[19] R. Alonso, M. Dhen, M. B. Gavela and T. Hambye, Muon conversion to electron in nuclei in type-I seesaw models, [arXiv:1209.2679 [hep-ph]]. 